\title{
Enumeration of non-positive planar trivalent graphs
}

\author{
Bruce W. Westbury
}

Received: 1 July 2005 / Accepted: 14 September 2006 /

Published online: 7 October 2006

(C) Springer Science + Business Media, LLC 2006

\begin{abstract}
In this paper we construct inverse bijections between two sequences of finite sets. One sequence is defined by planar diagrams and the other by lattice walks. In [10] it is shown that the number of elements in these two sets are equal. This problem and the methods we use are motivated by the representation theory of the exceptional simple Lie algebra $G_{2}$. However in this account we have emphasised the combinatorics.
\end{abstract}

Keywords Planar graphs $\cdot$ Lattice paths $\cdot$ Invariant tensors

\section{Introduction}

The aim of this paper is to give an enumeration of non-positive planar trivalent graphs. Here the graph is embedded in a disk and the number of boundary points is specified. This differs from other enumerations of planar trivalent graphs such as [16] or [4] in that neither the number of vertices nor the number of edges is specified. So if there were no further conditions then the number of graphs would be infinite. The extra condition that is imposed is that the graph is non-positive. This means that there is no internal face with less than six edges.

This problem arose in the work of Greg Kuperberg on the representation theory of the exceptional simple Lie algebra $G_{2}$ in [10]. In particular, one of the results of this paper is that the two sets we consider have the same numbers of elements. This is proved by showing that both sets are a basis of the same vector space.

Here, we give a bijective proof of this result. A bijective proof of the analogous result for $A_{2}$ is given in [7]. Moreover, the map from diagrams to words given below is constructed by the same method as the analogous map in this reference. However the

B. W. Westbury $(\square)$

Mathematics Institute, University of Warwick, Coventry CV4 7AL

e-mail: bww@maths.warwick.ac.uk 
construction of the inverse map that we give here is new. This construction is based on a diagram model for the crystal graph.

\section{Background}

The main result of this paper is the construction of inverse bijections between two sequences of finite sets. The two sets and the bijections are constructed by combinatorial methods and in writing this paper we have emphasised the combinatorial aspects. However both the original problem and the construction of the bijections are motivated by representation theory. The origins of the idea of using diagrams to represent invariant tensors are the Brauer algebras, spin networks and work of Cvitanovic. The advent of quantum groups and the applications to knot theory have made the use of diagrams more prevalent.

There are two combinatorial methods that have been introduced into representation theory and the study of invariant tensors; Littelmann paths and crystal graphs. The basic idea in this paper is to take the diagrams that have been introduced to study invariant tensors and to apply them to the crystal graph. The translation between these two points of view is given by the PRV theorem, [13, Theorem 2.1]. All of these methods can, in principle, be applied to other representations. In particular, these methods are applied to the spin representation of $\mathfrak{s o}(7)$ in [17].

In this paper we are studying the invariant theory of the seven dimensional fundamental representation of the exceptional simple Lie algebra of type $G_{2}$. The Lie algebra can be constructed as the derivation algebra of the octonions and the representation can then be taken to be the imaginary octonions. The weights and the dominant weights below are the usual weights and dominant weights of this Lie algebra. This representation has the (rare) property that all weight spaces are one dimensional. The set $S$ in (1) consists of the seven weights of this representation; moreover Fig. 6 (with the centre point of weight $(0,0))$ is the weight diagram of this representation.

The two finite sets we consider here each describe a basis for the vector space of invariants in the $n$-th tensor power of this representation. One basis is given by dominant Littelmann paths.

The Littelmann paths in this example are the seven paths drawn in Fig. 1. Then the vector space of highest weight vectors in the $n$-th tensor power of this seven dimensional representation has basis the set of concatenations of $n$ of these seven paths such that the concatenated path stays in the infinite wedge indicated by the shaded region in Fig. 2. The motivation for Definition 3.1 is that the lattice walks correspond to these paths. In particular the dimension of the space of invariant tensors in the $n$-th tensor power of this seven dimensional representation is the number of concatenations of $n$ of these seven paths which stay in this infinite wedge and which start and end at the origin.

The second method for constructing a basis of invariant tensors is to use crystal graphs. The crystal graph in this example is a directed graph with edges labelled by the simple roots where the vertices are the set $S$. The crystal graph is obtained from the crystal base. The crystal base and the crystal graph for this representation are given in [9]. These crystal graphs are then used in [11] to find the plactic monoid associated to this seven dimensional representation and also to find the crystal basis of 

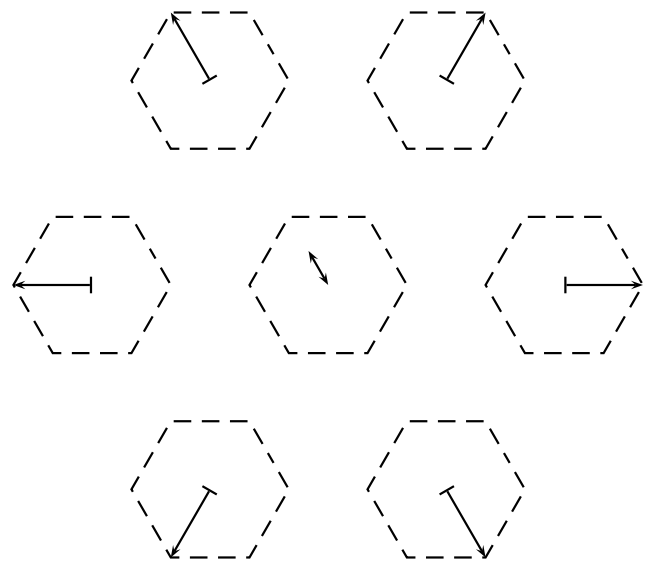

Fig. 1 Littelmann paths

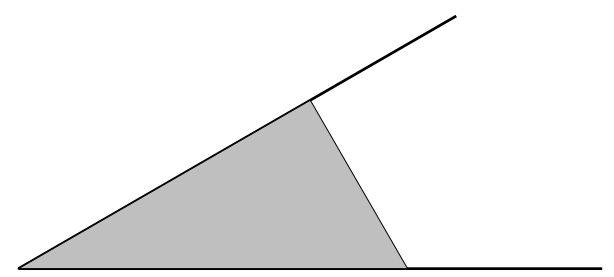

Fig. 2 Dominant weights

the irreducible representation of $U_{q}\left(G_{2}\right)$ associated to any dominant integral weight. In this example the vertices are parametrised by the weights and two weights are connected by an edge labelled by a simple root if the difference is the simple root. In this paper we do not make any use of the edges in the crystal graph and so they have been omitted.

The main innovation in this paper is to give the diagram model of the crystal graph as an alternative to the path model. So Fig. 1 is replaced by Fig. 8. Then we also want a diagram model for the tensor powers of the diagram model. This is described in Section 5.

Next we give the diagram model for crystal graphs of the tensor powers of the two dimensional fundamental representation of $\mathfrak{s l}(2)$. This is part of the graphical calculus in [3] and is included here for pedagogical purposes.

The diagram model for the crystal graph of this two dimensional representation is given by the two diagrams in Fig. 3.

Then the diagram models for the tensor powers of this crystal graph are drawn in the triangle in Fig. 4. A diagram consists of a finite number of non-intersecting arcs drawn in this triangle (with the endpoints in the boundary of the triangle). Furthermore every arc has at least one endpoint on the edge $X Y$. The length is the number of endpoints on the edge $X Y$. The height is the number of endpoints on the edge $Y A$ and the depth is the number of endpoints on the edge $A X$. 

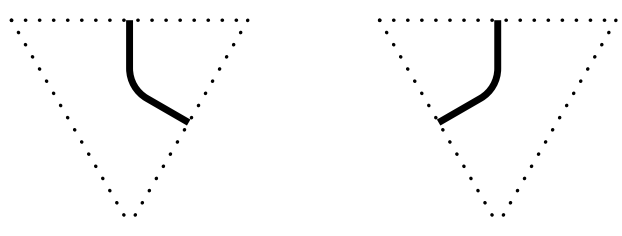

Fig. $3 \mathfrak{s l}(2)$ diagrams

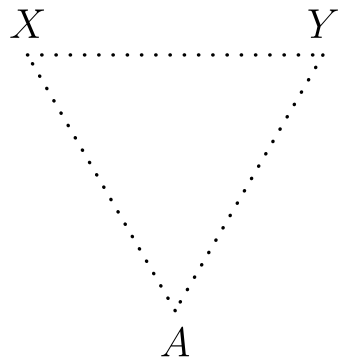

Fig. 4 Triangle

There is a bijection between the diagrams of length $n$ and words of length $n$ in the two letters ( and ). This bijection is given by associating one of these letters to each endpoint on the edge $X Y$. The rule is that given an endpoint we label it with (if the other endpoint of the arc is to the right on the edge $X Y$ or on the edge $Y A$ and we label it with ) if the other endpoint is to the left on the edge $X Y$ or on the edge $A X$.

Now we want to define the product of two of these diagrams. We require that the height and depth of the product is given by the rules for the tensor product of crystal graphs. This is illustrated in Fig. 10. It is clear that there is a unique way to fill in the diamond in Fig. 10 so that this rule is satisfied. This product makes the set of all diagrams into a graded monoid. This monoid is the free monoid on the two diagrams in Fig. 3. This is also the diagram model for the crystal graph of the tensor algebra of the fundamental representation.

The product of diagrams in Section 5 is not so clear and so we will now describe a more formal procedure for drawing the diagram associated to a word of length $n$ in the two generators. First we draw a grid; the grid for $n=4$ is shown in Fig. 5 .

Then to draw the diagram for a product of four generators; start by drawing the four generators in the four triangles along the top edge in Fig. 5. Now fill in the diamonds

Fig. 5 Grid 
by working down the grid. More precisely if the two triangles or two diamonds above a diamond have been filled in then that diamond can be filled in by choosing one of the four diamonds below:
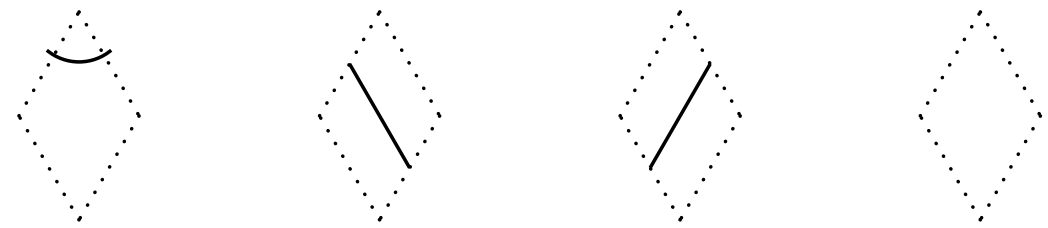

\section{Lattice walks}

In this section we will give the description of the lattice walks that will be related to the diagrams. We will use the terminology of weights which comes from representation theory.

A weight $\lambda$ is an ordered pair of integers. The set of weights is an abelian group under addition and is partially ordered. The partial order is given by saying $\left(a_{1}, b_{1}\right) \geq\left(a_{2}, b_{2}\right)$ if $a_{1} \geq a_{2}$ and $b_{1} \geq b_{2}$. A weight $\lambda$ such that $\lambda \geq 0$ is called a dominant weight.

Define the set $S$ to be the following set of seven weights

$$
S=\{(1,-2),(0,-1),(1,-1),(0,0),(0,1),(-1,1),(-1,2)\}
$$

Then the main definition of this section is

Definition 3.1. A lattice walk of length $n$ is a sequence of $n+1$ dominant weights $\left(\lambda_{0}, \lambda_{1}, \ldots, \lambda_{n}\right)$ such that $\left(\lambda_{i}-\lambda_{i-1}\right) \in S$ for $1 \leq i \leq n$. The sequence is also required to satisfy the additional condition that, for $1 \leq i \leq n$, if $\lambda_{i}=(a, 0)$ for some $a \geq 0$ then $\lambda_{i-1} \neq \lambda_{i}$.

If $\left(\lambda_{0}, \lambda_{1}, \ldots, \lambda_{n}\right)$ is a lattice walk then we say that the walk starts at $\lambda_{0}$ and ends at $\lambda_{n}$. Denote the set of lattice walks of length $n$ which start at $\lambda$ and end at $\mu$ by $W(\lambda, n, \mu)$. Also let the number of elements of $W(\lambda, n, \mu)$ be denoted by $w(\lambda, n, \mu)$.

Next we interpret a lattice walk as a walk in the triangular lattice. First we identify the set of weights with the vertices of the triangular lattice. This is an identification of abelian groups. The identification we use identifies the non-zero weights in $S$ with the regular hexagon of nearest neighbours of the origin in the triangular lattice. This is shown in Fig. 6.

Example 3.2. The following diagram has $w(0,5, \mu)$ written at position $\mu$.

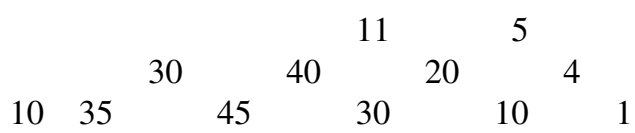




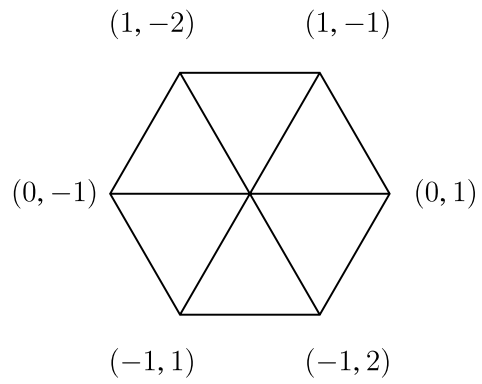

Fig. 6 Seven steps

Example 3.3. The following diagram has $w(0,6, \mu)$ written at position $\mu$.

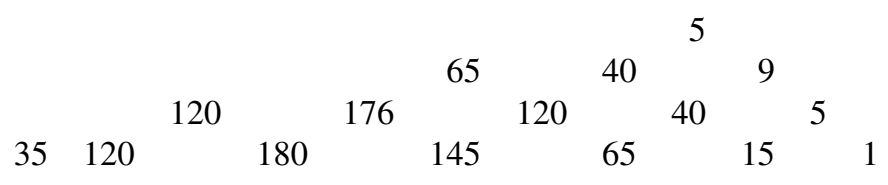

It is clear that the sets $W(\lambda, n, \mu)$ can be enumerated. Here we give numbers of the form $w(0, n, \mu)$. Let $X$ and $Y$ be the following Laurent polynomials in the indeterminates $x$ and $y$ :

$$
\begin{aligned}
X= & 1+x+x^{-1}+x^{-1} y+x y^{-1}+x^{-2} y+x^{2} y^{-1} \\
Y= & x-x^{-3} y^{2}+x^{-6} y^{3}-x^{-8} y^{3}+x^{-8} y^{2} \\
& -x^{-6}+x^{-3} y^{-2}-x y^{-4}+x^{4} y^{-5}-x^{6} y^{-5}+x^{6} y^{-4}-x^{4} y^{-2}
\end{aligned}
$$

Then the numbers $w(0, n, \mu)$ can be computed from the observation that if $\mu=$ $(a, b)$ then $w(0, n, \mu)$ is the coefficient of $y^{a} x^{b}$ in the Laurent polynomial $Y X^{n-1}$. The reason for this is that $X$ is the Weyl character of this representation; this is the generating function for the seven points in Fig. 6. The Weyl group of $G_{2}$ is the dihedral group of order twelve. Then $Y$ is the generating function for the orbit of $(0,1)$ under the action of the Weyl group with origin moved to $(-1,-1)$. This gives a regular polygon with twelve sides with centre at $(-1,-1)$. The signs are such that if two points are related by a reflection then they have different signs. The powers $X^{n}$ are the generating functions for sequences of steps of length $n$. Then the effect of multiplying by $Y$ is to impose the boundary conditions.

The sequence $a(n)=w(0, n, 0)$ which is the number of lattice walks of length $n$ which start and end at the origin is of particular interest. This sequence is given in [14] as sequence A059710. Equivalently $a(n)$ is the constant term in the expansion of $Y X^{n-1}$ where $X$ and $Y$ are the Laurent polynomials in (2). It follows from this description that the sequence $a(n)$ satisfies a finite recurrence relation with polynomial coefficients. Alex Mihailovs has proposed that this sequence satisfies the following recurrence Springer 
relation

$$
\begin{aligned}
(n+5)(n+6) a(n)= & 2(n-1)(2 n+5) a(n-1)+(n-1)(19 n+18) a(n-2) \\
& +14(n-1)(n-2) a(n-3)
\end{aligned}
$$

together with the initial conditions $a(0)=1, a(1)=0, a(2)=1$. The evidence for the proposal is that it gives the initial terms of the sequence and gives the correct asymptotics.

\section{Diagrams}

The main definition of this section is the following:

Definition 4.1. A diagram with $n$ boundary points consists of a disc with $n$ marked points on the boundary together with an embedded graph. The graph has $n$ vertices of valence one which are identified with the marked boundary points by the embedding and all other vertices of the graph have valency three.

A region is a connected component of the complement of the image of the graph in the disc.

Definition 4.2. A diagram is non-positive if every region of the disc which is bounded by edges of the graph is bounded by at least six edges of the graph.

Example 4.3. There are four non-positive diagrams with four boundary points. These are
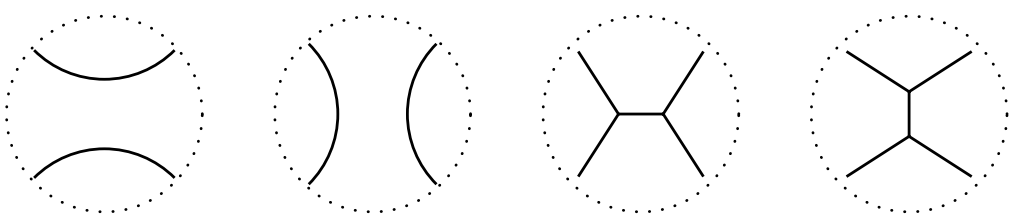

Although we will not make use of this result we first recall the argument from [10] which shows that if we specify the number of boundary points then there are only finitely many non-positive diagrams. This proof depends on the isoperimetric inequality given in [?].

Proposition 4.4. Let $n \geq 0$. Then there are finitely many non-positive diagrams with $n$ boundary points.

Proof: Consider a diagram whose graph is connected. Then the dual graph gives a triangulation of the disc. Take each triangle to be a Euclidean equilateral triangle with edge length 1 . Then this gives a polyhedral metric on the disc. Now if the planar graph is non-positive then this polyhedral metric has non-positive curvature. Hence the 
isoperimetric inequality is satisfied. Each triangle has area $\sqrt{3} / 2$ and the length of the boundary is $n$. Hence the isoperimetric inequality gives that there at most $n^{2} /(\pi \sqrt{3})$ triangles.

Next we recall a crucial definition from [10].

Definition 4.5. Assume we are given a diagram. Let $A$ and $B$ be two boundary points which are not marked points. Then a cut path from $A$ to $B$ is a path from $A$ to $B$ such that each component of the intersection with the embedded graph is either an isolated transverse intersection point or else is an edge of the graph.

The diagrams for these two cases are:
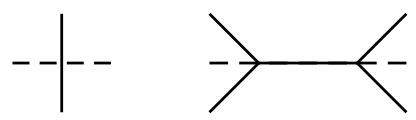

A cut path which crosses $b$ edges and contains $a$ edges is assigned the weight $(a, b)$. The weight $(a, b)$ is called dominant if $a \geq 0$ and $b \geq 0$. The weights are partially ordered by $\left(a_{1}, b_{1}\right)<\left(a_{2}, b_{2}\right)$ if $2 a_{1}+b_{1}<2 a_{2}+b_{2}$ or if $2 a_{1}+b_{1}=2 a_{2}+b_{2}$ and $b_{1}<b_{2}$. A cut path is minimal if there is no cut path with the same endpoints and with lower weight.

Definition 4.6. A triangular diagram is a non-positive diagram together with three points $A, X$ and $Y$ which are in the boundary of the disc but not marked points. Then we require that the edges $A X$ and $A Y$ are minimal cut paths.

We will draw a triangular diagram as in Fig. 4. The length of a triangular diagram is the number of marked points on the edge $X Y$.

Definition 4.7. A triangular diagram is reducible if there is a point $B$ inside the triangle (and not on the graph) such that there is a minimal cut path from $A$ to $X$ which passes through $B$, a minimal cut path from $A$ to $Y$ which also passes through $B$ and such that the the triangular diagram with vertices $B, X$ and $Y$ and edges given by these minimal cut paths from $B$ to $X$ and $B$ to $Y$ is a proper subdiagram.

A triangular diagram is irreducible if it is not reducible. The diagram for a reducible triangular diagram is given in Fig. 7. Note that since they are both minimal the paths $A X$ and $A B+B X$ have the same weight. Hence they are related by $H$-moves, see [10, Lemma 6.5]. For example, the seven diagrams in Fig. 8 are irreducible triangular diagrams of length one and Fig. 9 shows an irreducible triangular diagram of length two and a reducible triangular diagram of length one.

\section{Diagrams to paths}

In this section we construct a map $T(n) \rightarrow S^{n}$. 


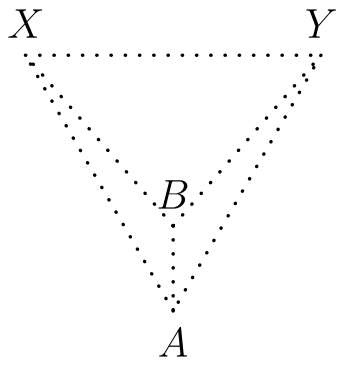

Fig. 7 Reducible diagram

Let $T(n)$ be the set of irreducible triangular diagrams of length $n$ and $S^{n}$ the set of words in $S$ of length $n$. Then first we construct a maps $T(n) \rightarrow S^{n}$ for $n \geq 0$. The construction is essentially the same as the construction of the analogous map in [7].

Choose a sequence of points in the edge $X Y,\left(X_{0}, X_{1}, \ldots, X_{n}\right)$, with $X=X_{0}$ and $Y=X_{n}$ such that no point in the sequence is a marked boundary point and such that for $1 \leq i \leq n$ the interval $\left(X_{i-1}, X_{i}\right)$ contains exactly one marked boundary point. Now for $0 \leq i \leq n$ let $\lambda_{i}$ be the weight of a minimal cut path with endpoints $A$ and $X_{i}$. Then the claim is that $\left(\lambda_{i}-\lambda_{i-1}\right) \in S$ for $1 \leq i \leq n$. This condition follows from the following:

Proposition 5.1. The irreducible triangular diagrams of length one are precisely the seven triangular diagrams in Fig. 8.

An isoperimetric inequality for sectors is given in [1]. It would be interesting to know if this isoperimetric inequality also holds for polyhedral metrics; and, if so,

$(0,2)$

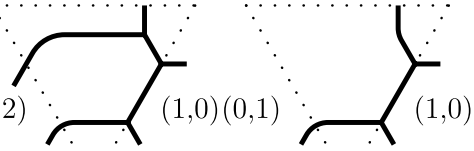

$(0,1)$<smiles>CCCCC</smiles>

$(0,0)(0,1)$
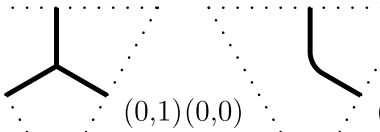

$(0,1)$

$(1,0)$

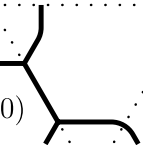

$(0,1)(1,0)$

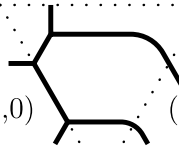

Fig. 8 Steps as triangles 

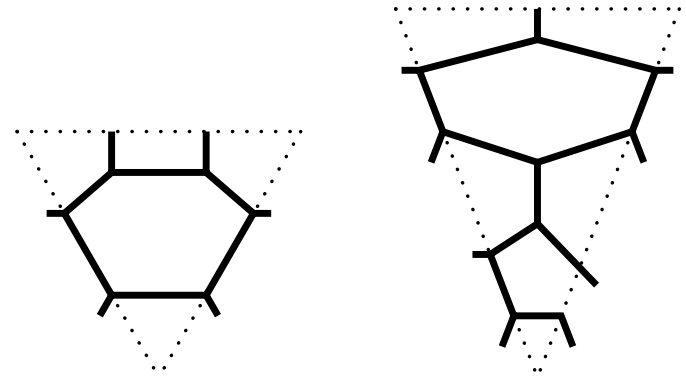

Fig. 9 Triangular diagrams

whether this implies that the number of irreducible triangular diagrams of length one is finite.

Proof: It is straightforward to check that each of the seven diagrams in Fig. 8 is an irreducible triangular diagram of length one. It remains to show that these are all such diagrams.

For the first observation assume that we are given an irreducible triangular diagram $A X Y$ and a point $B$ on the edge $A X$ but not on the graph. Then we can find a minimal cut path from $B$ to $Y$. Then the diagram $B X Y$ is a triangular diagram which may be reducible. If the diagram $B X Y$ is reducible then by [10, Lemma 6.6] there is a minimal cut path from $B$ to $Y$ of the same weight so that the triangular diagram $B X Y$ is irreducible.

It follows from this observation that if there is a counter example then there is a counter example such that the irreducible triangular diagram $B X Y$ is one of the seven triangular diagrams in Fig. 8 and such that the weight of the cut path $A B$ is either $(0,1)$ or $(1,0)$.

Now observe that in this situation the weight of $A Y$ is at most the sum of the weights of $A B$ and $B Y$; otherwise $A Y$ would not be a minimal cut path. Then the weight of $A Y$ is less than the sum of the weights of $A B$ and $B Y$; otherwise the triangular diagram $A X Y$ would be reducible.

There are only finitely many triangular diagrams with the following properties. The triangular diagram $B X Y$ is one of the seven triangular diagrams in Fig. 8; the weight of $A B$ is $(0,1)$ or $(1,0)$; the weight of $A Y$ is less than the sum of the weights of $A B$ and $B Y$.

To prove the proposition it is sufficient to check that none of these diagrams give a counter example.

Example 5.2. As a simple example we give the paths associated to the four diagrams in Example 4.3.

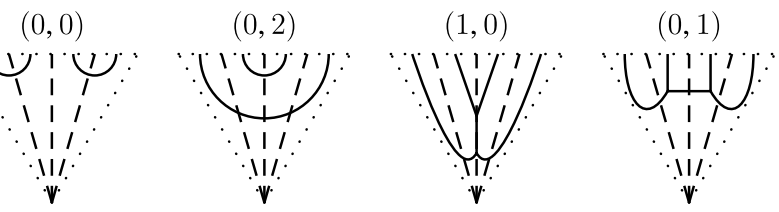


The weight on the top edge is the weight of the central cut path. The weights of the two sides of the triangle are $(0,0)$ in all four cases and the weights of the two intermediate cut paths are $(0,1)$ in all four cases. Taking the successive differences of these five weights gives a sequence of four elements of $S$ in each case. In each case the first element of the sequence is $(0,1)$ and the last element is $(0,-1)$.

\section{Paths to diagrams}

In this section we construct a map $S^{n} \rightarrow T(n)$. First we introduce a new type of edge. This will be drawn as a double edge. The Definition 4.1 (of a diagram) is modified to allow this new type of edge. The graph is still required to be trivalent but we also allow a vertex to be incident to two edges of the original type and to one of the new type. The Definition 4.5 (of a cut path) is also modified to allow a cut path to intersect any edge transversally. A cut path which crosses $b$ edges of the original type, contains $a$ edges (of the the original type) and crosses $c$ edges of the new type has weight $(a+c, b)$. This new type of edge is natural from the point of view of the representation theory. Given a word in $S^{n}$ we will describe how to construct a diagram which may include this double edge. Once this diagram has been constructed we eliminate the double edge using the following replacement; see [10, Section 4 (4) and Section 6.2]. This gives the element of $T(n)$.

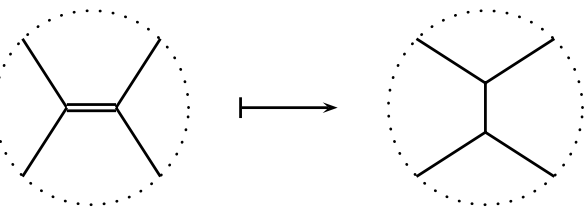

Construct a planar graph by taking the subgraph of the square lattice on the vertices $(i, j)$ such that $0 \leq i, j$ and $i+j \leq n$. Identify this subgraph of the square lattice with a grid as in Fig. 5 , where $(0,0)$ corresponds to the bottom vertex, $(0, n)$ to the top left corner and $(n, 0)$ to the top right corner. Then we label the edges of this graph by dominant weights. Label the edge from $(i, j)$ to $(i, j+1)$ by $D_{i, j}$ and label the edge from $(i, j)$ to $(i+1, j)$ by $H_{i, j}$. The labelling is constructed by induction on $n-i-j$.

The first stage in the construction of the diagram is to read the sequence of steps as a sequence of triangular diagrams using the correspondence in Fig. 11. This fills in the triangles in the top row of the grid in Fig. 5 and the corresponding labels on the edges of the triangles are the dominant weights $D_{i, j}$ and $H_{i, j}$ for $i+j=n-1$.

Then the remaining labels $D_{i, j}$ and $H_{i, j}$ are constructed by induction on $n-i-j$. These rules are the rules for the height and depth of the vertices of the tensor product of two crystal graphs. The tensor product of two crystal graphs was first defined in [5, Section 6] and other accounts are given in [15, Section 3] and [9, Section 4.4]. These fix a $k$ and determine the $k$-th components of dominant weights by applying the same rule that applies for $\mathfrak{s l}(2)$. The explicit rule for the $k$-th components is: If $D_{i+1, j-1}(k) \geq H_{i, j}(k)$ then put

$$
D_{i, j-1}(k)=D_{i+1, j-1}(k)-H_{i, j}(k) \quad H_{i, j-1}(k)=0
$$




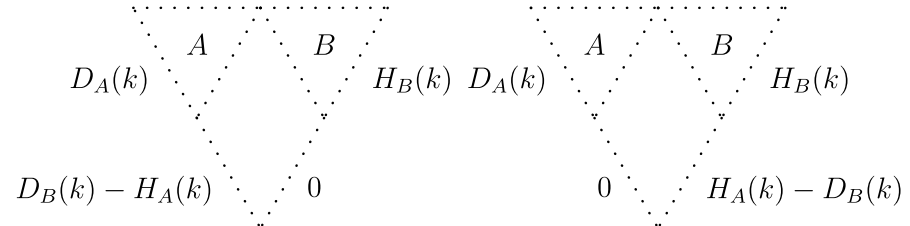

Fig. 10 Labelling the edges

and if $H_{i, j}(k) \geq D_{i+1, j-1}(k)$ then put

$$
D_{i, j-1}(k)=0 \quad H_{i, j-1}(k)=H_{i, j}(k)-D_{i+1, j-1}(k)
$$

This rule is shown on the triangular diagrams in Fig. 10.

At this point we have filled in the triangles in the top row of the grid in Fig. 5 and we have labelled all the edges in the grid by dominant weights. Next we give the rule for filling in each diamond. The top two edges of the diamond have been labelled by one of the four dominant weights

$$
\{(0,0),(0,1),(0,2),(1,0)\}
$$

This observation follows from an inductive argument. The basis of the induction is that all sides of all triangles in Fig. 11 are labelled by one of these four dominant weights. The inductive step is a consequence of the rule for constructing the labelling.

This gives sixteen labelled diamonds. Next we give a diagram for each of these sixteen labelled diamonds. The rule for completing the diagram is then that each diamond in the grid is filled in with the corresponding diamond diagram.

There are four symmetric diamonds which are also the diamonds where the weights on the top two edges are equal and the weights on the bottom two edges are zero. The

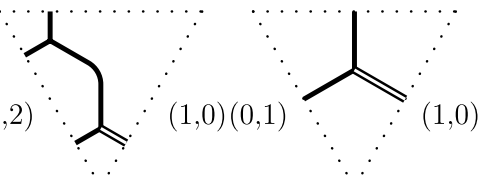

$(0,1)$

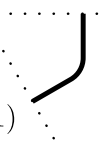

$(0,0)(0,1)$
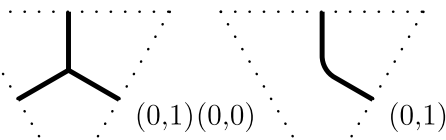

$(1,0)$

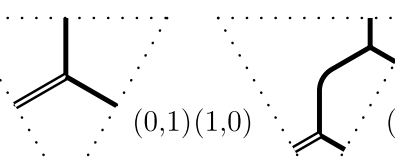

Fig. 11 Steps as triangles 
case in which all four weights are zero is an empty diamond. The other three diamonds are

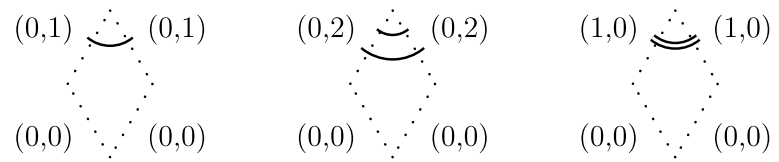

The other twelve diamonds come in pairs and we only give one member of each pair. The other member is obtained by reflection in a vertical line. There are three diagrams in which an oposite pair of edges have weight zero. These give the three diamonds
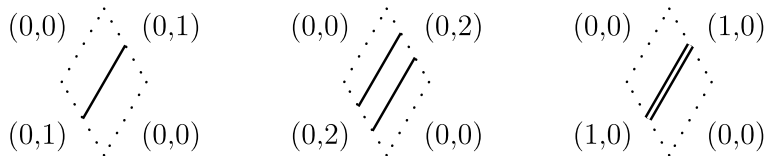

The remaining three diamonds are
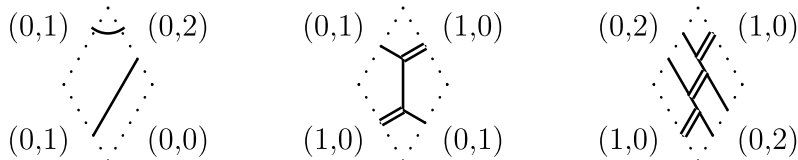

Example 6.1. If we draw the hexagon and take the sequence of dominant weights we get the top row below and then the second row is the sequence of six elements of $S$ obtained by taking successive differences.
$(0,0)$
$(0,1)$
$(1,0)$
$(0,2)$
$(1,0)$
$(0,1)$
$(0,0)$
$(0,1) \quad(1,-1) \quad(-1,2)$
$(1,-2) \quad(-1,1) \quad(0,-1)$

Then if we were given this sequence we can recover the hexagon. The first stage of the construction is to draw this as a sequence of six triangles. Then we find that the diagram completes when we fill in the first row of diamonds. This is shown in Fig. 12. Then substituting for the two double edges gives the hexagon.

The construction which associates a word to a diagram can be applied to a diagram which looks at first sight like an element of $T(n)$ but which may be positive or reducible.

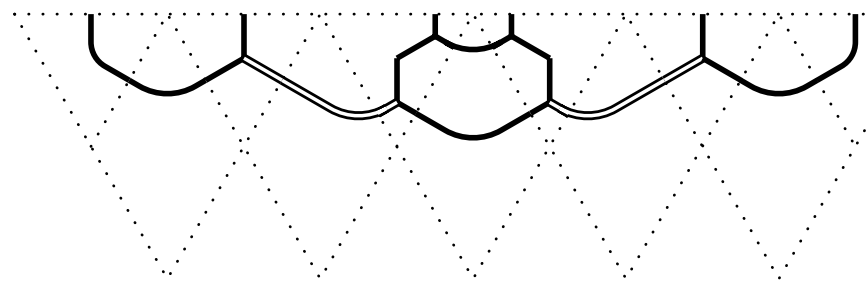

Fig. 12 Constructing the hexagon 
This will still give an element of $S^{n}$ and then we can construct an element of $T(n)$. This gives a projection map from the set of these more general diagrams to $T(n)$. The following two examples illustrate this.

Example 6.2. The square is positive and if we look at the cut paths we get the following diagram

$(1,0)$

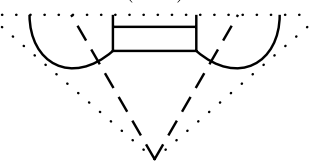

This gives the path labelled $(1,0)$ in Example 5.2.

Example 6.3. The pentagon is also positive and if we draw the diagram and take the sequence of dominant weights we get the top row below and then the second row is the sequence of five elements of $S$ obtained by taking successive differences.

$$
\begin{array}{lccccc}
(0,0) & (0,1) & (1,0) & (1,0) & (0,1) & (0,0) \\
(0,1) & (1,-1) & (0,0) & (-1,1) & (0,-1) &
\end{array}
$$

Note that this path does not satisfy the conditions of Definition 3.1 since we have $\lambda_{3}=(1,0)=\lambda_{4}$. The triangular diagram associated to this sequence is

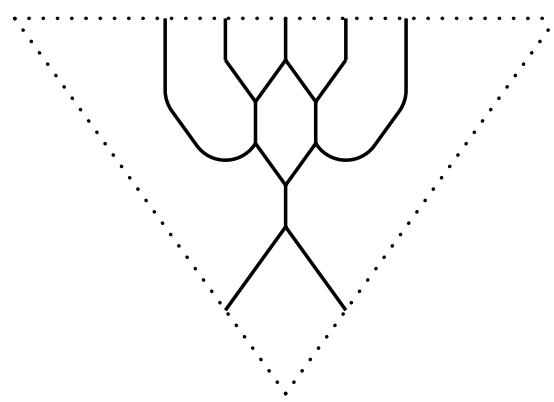

If we take the sequence of dominant weights associated to this diagram we get the sequence in the top row of $(2)$ with $(0,1)$ added to each term. The sequence of five elements associated to this is then the second row of (2). This corresponds to moving the starting point from $(0,0)$ to $(0,1)$ and this path does satisfy Definition 3.1.

It is possible to construct the diagram without introducing a double edge. The starting point is to fill in the triangles in the top line of the grid (in Fig. 5) using Fig. 8 (instead of Fig. 11). The labels on the edges of the grid are the same as before. Then for each of the sixteen diamonds we have a rule for extending the diagram to include the diamond. The reason we need rules for extending diagrams is that if we simply fill in the diamonds we may introduce a square, which is positive. If the diagram for filling in a diamond does not have a double edge then we fill in using the same diagram. The Springer 
remaining cases are covered by the four rules below (and their reflections in a vertical line). On the left we give the diamond before we extend and on the right the diamond after extending.
(1,0) $>>(1,0)$

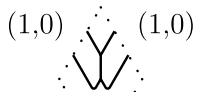
$(0,0) \because(0,0)$
$(0,0) \because(0,0)$
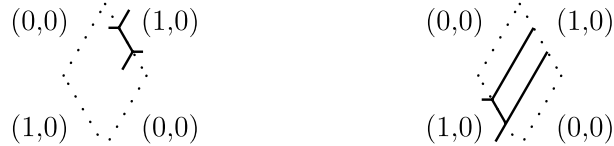
$(0,1) \therefore \nmid(1,0)$
$(1,0) \because(0,1)$

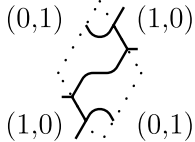
$\therefore>(1,0)$
$(1,0) \because(0,2)$

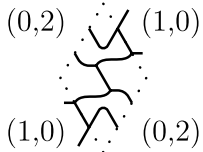

We can now state the main theorem of this paper.

Theorem 6.4. For all $n \geq 0$ these are inverse bijections between words in $S$ of length $n$ and irreducible triangular diagrams of length $n$.

An important feature of this construction is the following observation:

Lemma 6.5. Any increasing path which starts at the bottom vertex and follows the construction lines to the top edge of the diagram is a minimal cut path.

This observation shows that the diagram is non-positive since it shows that we have a minimal cut path from the bottom vertex to the top edge which passes through any internal region. Hence we have constructed a triangular diagram of length $n$. It remains to check that this is irreducible. This is the statement that the edges of the triangle are the unique minimal cut paths between their endpoints.

Then Lemma 6.5 also shows that if we start with a word construct the diagram and then derive a word that we recover the original word. In particular this shows that for $n \geq 0$, the map $T(n) \rightarrow S^{n}$ is surjective.

Next we show that, for $n \geq 0$, the map $T(n) \rightarrow S^{n}$ is injective. The proof is by induction on the length of the word. The basis of the induction is the case of length one which is proved in Proposition 5.1. Assume the result for words of length $n$. Let $w$ be a word of length $n+1$. Let $w_{i}$ be obtained by dropping the final step and $w_{f}$ be obtained by dropping the first step. Then these are both words of length $n$ and so by the inductive hypothesis have unique diagrams. 


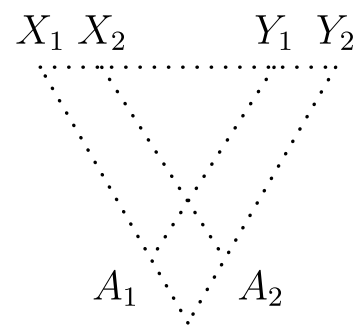

Fig. 13 Inductive step

Now assume we are given an irreducible triangular diagram which represents $w$ and draw this in the triangle in Fig. 13. Then there are points $X_{2}$ and $Y_{1}$ on the top edge so that $X_{1} Y_{1}$ and $X_{2} Y_{2}$ have length $n-1$. Now find a minimal cut path from $Y_{1}$ to the bottom vertex $A$. Then the diagram $A X_{1} Y_{1}$ is triangular but may be reducible. However, by [10, Lemma 6.6], there is a point $A_{1}$ so that the diagram in $A_{1} X_{1} Y_{1}$ is irreducible. This irreducible diagram is then the unique irreducible diagram which represents $w_{i}$. Similarly there is a point $A_{2}$ such that the diagram in $A_{2} X_{2} Y_{2}$ is the unique irreducible triangular diagram which represents $w_{f}$.

Thus the only part of the diagram that has not been filled in is the lowest diamond between the points $A_{1}$ and $A_{2}$. Thus the claim is that for each of the sixteen possible diamonds the rule we have given for filling it in is the unique rule that gives a nonpositive irreducible triangular diagram. For each of these sixteen diamonds, the claim can be checked using [10, Lemma 6.5].

This proves Theorem 6.4. Then for $n \geq 0$, these bijections restrict to bijections between the set of non-positive diagrams with $n$ boundary points and the set of lattice walks of length $n$ which start and end at the origin. There is a general principle which is illustrated in Example 6.3. Namely, given a word in $S^{n}$ construct the triangular diagram and let the dominant weight on the left edge be $D$. Then if we start at $D$ and take the path associated to the word then we get a lattice path; moreover $D$ is the lowest dominant weight with this property. In particular, lattice paths which start at the origin correspond to triangular diagrams whose left edge has weight $(0,0)$. Then the path ends at the weight of the right hand edge of the triangular diagram. So lattice paths which start and end at the origin correspond to triangular diagrams such that both sides have weight $(0,0)$.

One application of this is to the representation theory of the centraliser algebras of the tensor powers of the seven dimensional fundamental representation of $G_{2}$. Let $A(n)$ be the centraliser algebra of the $n$-th tensor power. From the diagram point of view, a basis of this algebra is the set of non-positive trivalent graphs drawn in a rectangle with $n$ boundary points on the top and bottom edges and no boundary points on the sides. Using cut paths which cross the rectangle we can construct cell ideals as defined in [?] and this shows that these algebras are cellular. The enumeration in this paper can be used to construct the cell representations. For each dominant weight $\mu$ we have the set of lattice paths $W(0, n, \mu)$. Then the bijection between $S^{n}$ and $T(n)$ restricts to a bijection between $W(0, n, \mu)$ and irreducible triangular diagrams of length $n$ whose left edge has weight $(0,0)$ and whose right edge has weight $\mu$. This set of diagrams may not be a basis for the cell representation because the sequence of 
transverse intersections and edges may not be the same for each diagram. However we can choose a fixed sequence. Then, given an irreducible triangular diagram whose right hand edge has weight $\mu$ but with a different sequence, there is a unique sequence of $H$-moves which changes the sequence on the right edge to the given sequence. This gives a reducible triangular diagram. These diagrams are a basis for the cell representation.

\section{References}

1. H. Bahn and S. Hong, "Isoperimetric inequalities for sectors on surfaces," Pacific J. Math. 196(2) (2000), 257-270.

2. S. Doty, "Presenting generalized q-Schur algebras," Represent. Theory 7 (2003), 196-213 (electronic), arXiv:math.QA/0305208.

3. I.B. Frenkel and M.G. Khovanov, "Canonical bases in tensor products and graphical calculus for $U_{q}\left(\mathfrak{s l}_{2}\right)$," Duke Math. J. 87(3) (1997), 409-480.

4. Z. Gao and N.C. Wormald, "Enumeration of rooted cubic planar maps," Ann. Comb. 6(3-4) (2002), 313-325.

5. M. Kashiwara, "Crystalizing the $q$-analogue of universal enveloping algebras," Comm. Math. Phys. 133(2) (1990), 249-260.

6. M. Kashiwara, "On crystal bases of the $q$-analogue of universal enveloping algebras," Duke Math. J. 63(2) (1991), 465-516.

7. M. Khovanov and G. Kuperberg, "Web bases for sl(3) are not dual canonical," Pacific J. Math. 188(1) (1999), 129-153, arXiv:q-alg/9712046.

8. S.-J. Kang, M. Kashiwara, K.C. Misra, T. Miwa, T. Nakashima, and A. Nakayashiki, "Affine crystals and vertex models," in Infinite Analysis, Part A, B (Kyoto, 1991), volume 16 of Adv. Ser. Math. Phys., World Sci. Publishing, River Edge, NJ, 1992, pp. 449-484.

9. S.-J. Kang and K.C. Misra, "Crystal bases and tensor product decompositions of $U_{q}\left(G_{2}\right)$-modules," $J$. Algebra 163(3) (1994), 675-691.

10. G. Kuperberg, "Spiders for rank 2 Lie algebras," Comm. Math. Phys. 180(1) (1996), 109-151, arXiv:qalg/9712003.

11. C. Lecouvey, "Schensted type correspondence for type $G_{2}$ and computation of the canonical basis of a finite dimensional $U_{q}\left(G_{2}\right)$-module", arXiv:math.CO/0211443.

12. P. Littelmann, "A plactic algebra for semisimple Lie algebras," Adv. Math. 124(2) (1996), 312-331.

13. K.R. Parthasarathy, R. Ranga Rao, and V.S. Varadarajan, "Representations of complex semi-simple Lie groups and Lie algebras," Ann. of Math. (2) 85 (1967), 383-429.

14. N.J. Sloane, "The on-line encylopedia of integer sequences", 2006. http://www.research.att.com/njas/ sequences/.

15. J.R. Stembridge, "Combinatorial models for Weyl characters," Adv. Math. 168(1) (2002), 96-131.

16. W.T. Tutte, "A census of planar triangulations," Canad. J. Math. 14 (1962), 21-38.

17. B.W. Westbury, "Invariant tensors for the spin representation of $\mathfrak{s o}(7)$ ", arXiv:math.QA/0601209.

18. S. Yamane, "Perfect crystals of $U_{q}\left(G_{2}^{(1)}\right)$," J. Algebra 210(2) (1998), 440-486, arXiv:q-alg/9712012. 